NASA/CR-1998-206902

ICASE Report No. 98-1

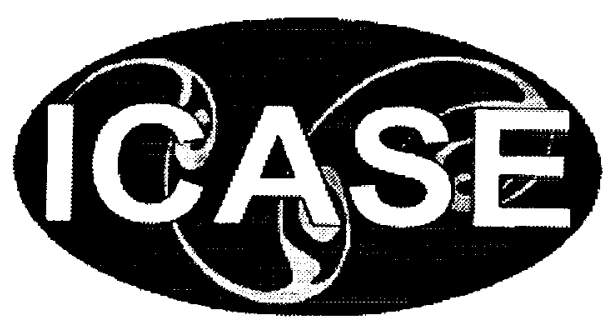

\title{
Some Results Relevant to Statistical Closures for Compressible Turbulence
}

\section{J. R. Ristorcelli}

ICASE

Institute for Computer Applications in Science and Engineering

NASA Langley Research Center

Hampton, VA

Operated by Universities Space Research Association

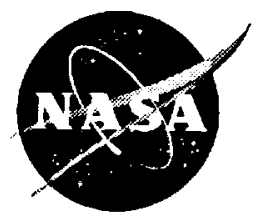

National Aeronautics and

Space Administration

Langley Research Center

Hampton, Virginia 23681-2199

Prepared for Langley Research Center under Contract NAS1-19480

January 1998 
Available from the following:

NASA Center for AeroSpace Information (CASI) 800 Elkridge Landing Road

Linthicum Heights, MD 21090-2934 (301) 621-0390
National Technical Information Service (NTIS) 5285 Port Royal Road

Springfield, VA 22161-2171

(703) 487-4650 


\title{
SOME RESULTS RELEVANT TO STATISTICAL CLOSURES FOR COMPRESSIBLE TURBULENCE
}

\author{
J. R. RISTORCELLI*
}

\begin{abstract}
For weakly compressible turbulent fluctuations there exists a small parameter, the square of the fluctuating Mach number, that allows an investigation using a perturbative treatment. The consequences of such a perturbative analysis in three different subject arcas are described: 1) initial conditions in direct numerical simulations, 2) an explanation for the oscillations seen in the compressible pressure in the direct numerical simulations of homogeneous shear, and 3) for turbulence closures accounting for the compressibility of velocity fluctuations.

1) Initial conditions consistent with small turbulent Mach number asymptotics are constructed. The importance of consistent initial conditions in the direct numerical simulation of compressible turbulence is dramatically illustrated: spurious oscillations associated with inconsistent initial conditions are avoided, and the fluctuating dilatational field is some two orders of magnitude smaller for a compressible isotropic turbulence. For the isotropic decay it is shown that the choice of initial conditions can change the scaling law for the compressible dissipation.

2) A two-time expansion of the Navier-Stokes equations is used to distinguish compressible acoustic and compressible advective modes. A simple conceptual model for weakly compressible turbulence -- a forced linear oscillator - is described. It is shown that the evolution equations for the compressible portions of turbulence can be understood as a forced wave equation with refraction. Acoustic modes of the flow can be amplified by refraction and are able to manifest themselves in large fluctuations of the compressible pressure.

3) The consequences of a small turbulent Mach number expansion for the closure of two covariances appearing in the kinetic energy equation, the pressure-dilatation and the dilatational dissipation, are investigated. Comparisons with different models and a discussion of the results in the context of the homogeneous shear is given. In agreement with observations of DNS of compressible turbulence, the dilatational covariances can not account for the large reductions in the growth of compressible shear layers.
\end{abstract}

Key words. Dilatation, compressible turbulence, turbulence modeling.

Subject classification. Fluid Mechanics

1. Introduction. The phrase weakly compressible describes the turbulence relevant to many aerodynamic applications. Several interesting insights into the nature of compressible turbulence can be found by investigations of the weakly compressible limit: the weakly compressible limit allows a perturbative treatment. Such weakly compressible investigations are relevant to compressible shear layers encountered in practical applications.

For weakly compressible fluctuations the turbulent Mach number squared is small, $M_{t}^{2} \ll 1$, and serves as a small parameter. Here $M_{t}=u_{c} / c ; u_{c}$ is a fluctuating velocity scale and $c$ is the local mean speed of sound. The turbulent Mach number reflects the weakly compressible nature of the turbulence - the

*ICASE, M/S 403, NASA Langley Research Center, Hampton, VA, 23681-0001; email: jrrjr@icase.edu. This research was supported by the National Aeronautics and Space Administration under NASA Contract No. NAS1-19480 while the author was in residence at the Institute for Computer Applications in Science and Engineering (ICASE), M/S 403, NASA Langley Research Center, Hampton, VA 23681-0001. 
mean flow itself may be highly supersonic. An analytical development for the covariances of the fluctuating dilatation suitable for shear flows has been given in Ristorcelli (1997). This article describes the implications of such an analysis for the initial conditions in the direct numerical simulation of turbulence as discussed in Ristorcelli and Blaisdell (1997). This article also reports an extension of that analysis that can explain the peculiar oscillations seen in the DNS of homogeneous shear. In the process a simple conceptual model - the forced linear oscillator - for compressible turbulence is derived. This article also explores, in the context of the DNS, representations for terms requiring closure in the kinetic energy equation.

The primary subject of this article, given that it is to appear in the proceedings of a conference devoted to turbulence modeling, is a summary of recent advances made in the understanding of the effects of compressibility as related to turbulence models for engineering flows. To this end a substantial portion of space is spent understanding numerically generated data bases which are used to develop turbulence models. This is done to insure that the physics of the numerically simulated flow is consistent with the enginecring problem and thus constitutes a relevant data base for statistical closures relevant to engineering flows.

This article is divided into three separate sections.

Section 2 treats initial conditions for the DNS of compressible turbulence. It is shown that as a consequence of a finite turbulent Mach number there are finite non-zero density, tempcrature and dilatational fluctuations. These fluctuations are, to lowest order, specified by the incompressible field. These results are relevant to initial conditions on thermodynamic quantities employed in DNS of compressible turbulence that are started using incompressible fluctuating velocity fields. Use of these initial conditions gives rise to a smooth development of the flow, in contrast to cases in which these fields are specified arbitrarily (or set to zero). For the isotropic decay it is shown that the choice of the initial conditions appears to change the scaling law for the compressible dissipation. It will be seen that, for the isotropic decay, the well accepted $M_{t}^{2}$ scaling for the compressible dissipation appears to be an artifact of an evanescent wave field set up by the initial conditions.

In Section 3 a simple conceptual model for weakly compressible turbulence is described: the evolution equations for the compressible portions of turbulence in the presence of a homogeneous shear are seen to obey a forced wave equation with refraction. The forcing and refraction effects are a function of the shear rate as indicated by a gradient Mach number.

In Section 4 the results of the modeling of Ristorcelli (1997) are explored. It is shown that $M_{t}^{4}$ scaling for the compressible dissipation is consistent with the DNS of compressible turbulence results with the appropriate initial conditions. A scaling of $M_{t}^{2}$ for the compressible dissipation is consistent with the DNS if "inconsistent" initial conditions are used. A verification of the scalings and comparision with other models for the pressure-dilatation is also conducted.

2. Initial Conditions. For turbulence with finite turbulent Mach number there is a finite effect of compressibility. A methodology, consistent with finite Mach number asymptotics, for generating initial conditions for the fluctuating pressure, density, temperature, and dilatational velocity fields is described. Relationships between diverse thermodynamic quantities appropriate to weakly compressible turbulence are derived. The phrase consistent initial conditions is used to denote initial conditions that are suggested by low turbulent Mach number asymptotics.

2.1. Single time perturbation analysis. Ristorcelli (1997) has conducted a small Mach number expansion of the compressible Navier-Stokes equations. Some of those results, as they are relevant to the present subject, are now briefly sketched. The problem of compressible turbulence can be viewed as a singular perturbation. The inner problem is related to the acoustic source problem with time and length 
scales based on the turbulence time and length scales, $\ell / u_{c}$ and $\ell$. Here $u_{c}$ is the turbulence velocity scale and $\ell$ is the turbulence integral length scale. The outer problem is the acoustic propagation problem, which in the low $M_{t}^{2}$ limit has time and length scales $\lambda / c=\ell / u_{c}$ and $\lambda=\ell / M_{t}$. The inner expansion is relevant to the compressible turbulence modeling problem of acoustically compact flows, Ristorcelli (1997). The outer problem is not relevant to the problem of compressible turbulence for a compact fields, Ristorcelli (1997).

The compressible Navier-Stokes equations in a form convenient for weakly compressible high Reynolds number turbulence are

$$
\begin{aligned}
\rho_{, t}+u_{k}^{*} \rho_{, k} & =-(1+\rho) u_{p, p}^{*} \\
(1+\rho)\left[u_{i, t}^{*}+u_{k}^{*} u_{i, k}^{*}\right]+\frac{c_{\infty}^{2}}{\gamma} p_{, i} & =0 \\
p-\gamma \rho & =\frac{1}{2} \gamma(\gamma-1) \rho^{2} \\
\rho_{, t t}-\frac{c_{\infty}^{2}}{\gamma} p_{, j j} & =\left((1+\rho) u_{i}^{*} u_{j}^{*}\right)_{, i j} .
\end{aligned}
$$

The last equation, a wave equation, is derived from continuity and momentum. The velocity field is decomposed according to $u_{i}=v_{i}+\epsilon^{2} w_{i}+\ldots$, where $v_{i}$ represents the solenoidal velocity field and the the small parameter is $\epsilon^{2}=\gamma M_{t}^{2}$, where $M_{t}=u_{c} / c$. Thus $d=u_{i, i}=\epsilon^{2} w_{i, i}+\ldots$. The thermodynamic variables are decomposed according to a perturbation about a mean state, $(P, \bar{\rho}, T)$, thus $p^{*}=P(1+p), \rho^{*}=\bar{\rho}(1+\rho)$ and $T^{*}=T(1+\theta)$. Perturbation series of the form $p=\epsilon^{2}\left[p_{1}+\epsilon^{2} p_{2}+\ldots\right]$ are assumed. To lowest order

$$
\begin{aligned}
v_{i, t}+v_{p} v_{i, p}+p_{1, i} & =0 \\
v_{i, i} & =0 \\
p_{1, j j} & =-\left(v_{i} v_{j}\right)_{, i j} \\
\rho_{1} & =\frac{1}{\gamma} p_{1} \\
\theta_{1} & =\frac{\gamma-1}{\gamma} p_{1} .
\end{aligned}
$$

The zeroth-order problem is the incompressible problem. The fluctuating pressure is not an independent variable but is set by the solenoidal velocity fluctuations and which also produces the density and temperature fluctuations. On the inner scales the incompressible pressure fluctuations dominate the pressure field. This pressure is sometimes called the "pseudo-pressure" in contradistinction to the propagating pressure associated with the sound field. The last two equations come from the ideal gas law. In light of the homogeneous shear flow simulations of Blaisdell et al. $(1991,1993)$ (the fluctuations were seen to follow a polytropic gas law with coefficient close to the adiabatic value), the adiabatic case is treated.

The next order expansion for the continuity equation produces a diagnostic relation for the fluctuating dilatation,

$$
-\gamma d=p_{1, t}+v_{k} p_{1, k} .
$$

The dimensional equivalents of the above equations, using $\rho_{1}=\rho^{\prime} / \bar{\rho}, \theta_{1}=\theta^{\prime} / T, p_{1}=p^{\prime} / P$ are

$$
\begin{aligned}
\rho^{\prime} & =\frac{1}{c^{2}} p^{\prime} \\
\theta^{\prime} & =\frac{\gamma-1}{\gamma R \bar{\rho}} p^{\prime} \\
-\gamma P d^{\prime} & =p^{\prime}{ }_{, t}+v_{k} p^{\prime}{ }_{, k},
\end{aligned}
$$


where $p^{\prime}$ is the solenoidal pressure fluctuation. For finite turbulent Mach number there are unique specified finite fluctuations of density, temperature, dilatation and pressure.

Obtaining an initial condition for the compressible portion of the velocity, $w_{i}$, which reflects the relation (11) is possible, Ristorcelli and Blaisdell (1997). The fluctuating pressure $p_{1}$ needed in (11) is obtained from the Poisson equation (7), and its time derivative can be found by taking the time derivative of (7) and substituting for the time derivative of the velocity from (5). This gives a Poisson equation for $p_{1, t}$,

$$
\left(p_{1, t}\right),_{j j}=2\left[\left(v_{k} v_{i, k}+p_{1, i}\right) v_{j}\right]_{, i j}
$$

Solving the two Poisson equations (7) and (12), the dilatation is found from (10). For homogeneous turbulence the dilatational velocity can be found from the dilatation by working in Fourier space. As the dilatational velocity, $w_{i}$, is irrotational the Fourier coefficients of the velocity are aligned with the wavevector, $\vec{k}$, and they can be found from

$$
\widehat{w}_{j}=-i \frac{k_{j}}{k^{2}} \widehat{d}
$$

The dilatational velocity $w_{i}$ is then combined with the solenoidal velocity $v_{i}$ to obtain the full initial velocity field. This set of initial conditions, (11) and(13), will called the consistent (or pseudo-sound) initial conditions.

2.2. Simulations and Results. In order to determine the effectiveness of the new initial condition method, direct numerical simulations of decaying isotropic turbulence are carried out with three different types of initial conditions, Ristorcelli and Blaisdell (1997). Simulations were performed with initial turbulent Mach numbers $M_{t}=0.231,0.115$, and 0.058 . The initial turbulent Reynolds number $R_{T}=\bar{\rho} q^{4} / \varepsilon \nu=200$ where $q^{2}=\left\langle\rho u_{i} u_{i}\right\rangle / \bar{\rho}$, and $\varepsilon$ is the dissipation rate of turbulent kinetic energy per unit volume. The mean density gradient is zero. For the present analysis and simulations the Reynolds and Favre averages are the same. Details about the simulation method can be found in Blaisdell et al. (1991).

1. The first type of initial conditions, $\mathrm{IC} 1$, has zero density and pressure fluctuations. The velocity field is solenoidal.

2. The second type of initial conditions, IC2, has zero density fluctuations; the pressure is found by solving the Poisson equation (7). The velocity field is again solenoidal.

3. The third type of initial conditions, IC3, the fluctuating pressure field is found by solving the Poisson equation (7). The relations (11) determine the density and dilatational fluctuations from (7). The dilatation is used to determine the dilatational velocity from (13).

2.2.1. Magnitude of the dilatation. Data from the $M_{t}=0.231$ run has been chosen to illustrate the effect of the different initial conditions. Figure 1 shows the development of the variance of the dilatation, $\langle d d\rangle$. It is clear from the figure that there are sizeable oscillations near the origin for the first two initial conditions as the flow seeks to adjust to "inconsistent" initial conditions. This is not the case for the pseudosound initial conditions, IC3. Also interesting to note is the fact that the variance of the dilatation for IC1 is one order of magnitude larger than IC3. In general, at lower $M_{t}$ this difference is more pronounced; at $M_{t}=0.058$ the difference is two orders of magnitude. This excess dilatation is associated with an evanescent wave field, see $\S 3$, set up by an inappropriate choice of initial conditions and is not the dilatation due to the compressible turbulence field.

An intuitive argument can be given to explain the behavior seen in Figure 1. One might speculate that there is a wave field generated by the inconsistency between the pressure, density, temperature, and 
dilatational fields whose decay rate is far slower than the turbulence decay. This gives rise to a background evanescent acoustic radiation, for a homogeneous flow, that lasts the course of the simulation. For IC1 the fluctuating pressure field is set to zero. This can be viewed as a combination of the incompressible pressure satisfying (7) plus an acoustic pressure field which exactly cancels the incompressible pressure field - so that the initial field is zero. As time evolves the two pressure fields become decorrelated, giving rise to large acoustic pressure and dilatation fluctuations. For IC2 the pressure field is correct; however, there is no dilatation field. Again this can be viewed as a combination of the dilatation found from (7) plus an acoustic dilatation field that exactly cancels this. As the flow evolves away from the initial conditions, the two dilatation fields become decorrelated so that one is left with the correct dilatation plus acoustic fluctuations of dilatation and pressure associated with the slowly decaying evanescent field. The evolution of the pressure variance and $\langle p d\rangle$ (not shown here, Blaisdell (1996)) corroborates this picture.

2.2.2. Scaling of the dilatation. Figure 2, provided by Professor G.A. Blaisdell, is a very rich figure with many implications. Figure 2 is a graphic indication of the importance of initial conditions; it indicates the scaling of the dissipation with the turbulent Mach number.

The figure describes the isotropic decay conducted for three different initial turbulent Mach numbers. The three different initial conditions are seen to produce two different scalings for the compressible dissipation or, equivalently, the variance of the dilatation. For the consistent initial conditions the dilatation is seen to have $M_{t}^{4}$ scaling. This is indicated by the upper solid line. For the two sets of "inconsistent" initial conditions an $M_{t}^{2}$ scaling, as indicated by the lower dashed lines, is seen. The $M_{t}^{2}$ scaling is the scaling usually agreed on for some models of the compressible dissipation - it is a scaling that is arrived at from observations of DNS with inconsistent initial conditions.

2.3. Conclusions. It has been shown that as a consequence of a finite turbulent Mach number there are finite non-zero density, temperature and dilatational fluctuations. These fluctuations are, to lowest order, specified by the incompressible field. These results are relevant to initial conditions on thermodynamic quantities employed in DNS of compressible turbulence that are started using incompressible fluctuating velocity fields. A potential practical consequence of the present results is a reduction in the amount of computational effort spent adjusting to transients associated with the relaxation from arbitrary initial conditions.

The major point is that the initial conditions on the fluctuating thermodynamic variables of density, temperature, pressure, and dilatation should not be arbitrarily specified in the DNS of compressible turbulence. To lowest order these fluctuations, whose nature is connected to the underlying fluctuating vortical turbulence field, are generated by the pressure field associated with the divergence-free portion of the vortical motions. The methodology for generating initial conditions presented here allows the flow to develop more naturally.

The significance of the initial conditions is likely to depend on the type of flow considered; for example the effects described above are likely to be much less evident in a homogeneous shear in as much as the final $M_{t}$ substantial exceeds the initial $M_{t}$. Nonetheless, one is led naturally to speculate about the nature and relevance of simulations that start from arbitrary initial conditions. There are surely a large number of interesting studies in which one can study the relaxation from diverse arbitrary initial conditions. It is also possible to argue that they have practical value. However, the strongest argument, given the current engineering problems and the lack of knowledge regarding the effects of compressibility, can be made for the initial conditions in which the density, temperature, pressure, and dilatational fields are related to the underlying local turbulence field that is the source of the fluctuations. This seems better than an arbitrary guess at initial conditions that may or may not have physical relevance or meaning. 
3. Two-timing compressible turbulence. The present discussion is limited to a physical understanding of the effects of compressibility as arrived at mathematically. A two-time expansion of the compressible Navier-Stokes equations is used to investigate the compressible modes of a turbulent flow. Such a two-time scale analysis sheds substantial insight on the nature of compressibility.

The present treatment of the problem cuntrasts to that given by Ristorcelli (1997). That treatment, in which the the small parameter was also the iurbulent Mach numbers, used a single time expansion. The single time expansion was a consequence of nondimensionalizing time with a time scale characteristic of the turbulence, the eddy turnover time. Such an analysis is suitable for statistical closure that evolves on a nonlinear advective time scale. Here the investigation is directed towards distinguishing quantities that evolve on a fast time scale from those evolving on a slow time scale. It will be seen that the quantities evolving on the fast time, as can be assessed by their evolution equations, cannot be understood as turbulence. The fast time equations suggest that it is useful to interpret the fast time solution as an evanescent wave field.

It is appropriate to indicate the observations that motivate the present analysis. The experimental data that suggested this investigation is the DNS of the homogeneous compressible shear as might be seen in Blaisdell, Mansour and Reynolds (1991, 1993), Sarkar, Erlebacher and Hussaini (1991), or Sarkar (1992). Figure 3 is the pressure-dilatation from Blaisdell's recent simulations; the instantaneous ensemble averaged as well as the time averaged pressure-dilatation time history is shown. The initial conditions follow those outlined in Ristorcelli and Blaisdell (1997). Even with the initial conditions suggested by a single-time small turbulent Mach number expansion there is a build up of rapidly varying fluctuations in the pressuredilatation. This is also seen in the earlier simulations by Sarkar (1992); in Sarkar's (1992) simulations the oscillations occur earlier and this is consistent with the choice of initial conditions. The oscillations in the instantaneous pressure-dilatation exhibits a time scale characteristic of the sound crossing time over an eddy, $\ell / c$, as can be estimated from the DNS. The cvolution of the time averaged trace evolves on a slower time scale, the eddy turnover time, $\ell / u_{c} \sim k / \varepsilon$. The appearance of these two disparate time scales suggest that a two-time analysis of the compressible DNS may be appropriate to further understand the nature of the physics. Consider a characteristic fluctuating velocity, $u_{c}$, say related to the kinetic energy of the turbulence $k, u_{c}^{2}=\frac{2}{3} k$. The ratio of these two time scales is the fluctuating Mach number:

$$
M_{t}=\frac{u_{c}}{c} .
$$

If it is assumed that the ratio of the eddy crossing time to the turnover time is small, $\frac{\ell}{u_{c}}>>\frac{\ell}{c}$, (or equivalently the Mach number is small) a multi-scale expansion of the Navier-Stokes equations will produce a useful model. The small parameter in the two-time problem is now $\epsilon=M_{t}$. In the single time problem the appropriate small parameter was $M_{t}^{2}$. In the present development, as will become clear, the fluctuating Mach number plays two unique roles: It indicates the relative time scales of advection versus propagation in this role it appears to the first power. It is also a measure of the dynamical effects of the compressibility of the fluctuating field - in this role it appears to the second power.

3.1. Two-timing: analysis. A set of equations describing and distinguishing the relevant aspects of the flow physics is now derived. The procedure is straightforward and standard for those familiar with perturbation methods. Perturbing about a reference state, $\left(p_{\infty}, \rho_{\infty}\right)$, the dimensional pressure, density and velocity are taken as

$$
\begin{aligned}
& p^{*}=p_{\infty}(1+p) \\
& \rho^{*}=\rho_{\infty}(1+\rho) .
\end{aligned}
$$




$$
u_{i}^{*}=u_{c}\left(v_{i}+\phi_{w} w_{i}\right)
$$

where

$$
\begin{aligned}
& p=\phi_{0}\left(p_{s}+\phi_{1} p_{c}\right) \\
& \rho=\phi_{0}\left(\rho_{s}+\phi_{1} \rho_{c}\right) .
\end{aligned}
$$

The dependent variables are now all nondimensional: $\left[v_{i}, w_{i}, p_{s}, p_{c}, \rho_{s}, \rho_{c}\right]$ are in units of $\left[u_{c}, p_{\infty}, \rho_{\infty}\right]$. These forms of the dimensional variables are then inserted into the compressible equations, (1), (2), (3) of $\S 2$. The leading order problem, as in $\S 2.1$, is described by equations used for a solenoidal velocity field with characteristic time, length and velocity scales $\left[\frac{\ell}{u_{c}}, \ell, u_{c}\right]$,

$$
\begin{aligned}
v_{p, p} & =0 \\
v_{i, t}+v_{k} v_{i, k}+p_{s, i} & =0 \\
p_{s, j j} & =-\left(v_{i} v_{j}\right)_{, i j} \\
\gamma \rho_{s} & =p_{s} .
\end{aligned}
$$

Note that the leading order density fluctuations are given by $p_{s}$. The subscript " $s$ " is understood to indicate solenoidal as the field $v_{i}$ has zero divergence. The gauge function $\phi_{0}=\gamma M_{t}^{2}$ in order to obtain a meaningful balance with pressure.

The compressible portion of the problem, $\left[p_{c}, \rho_{c}, w_{i}\right]$, is described by the following set of equations:

$$
\begin{aligned}
{\left[1+\phi_{0}\left(\rho_{s}+\phi_{1} \rho_{c}\right)\right]\left[w_{i, t}+v_{k} w_{i, k}+w_{k} v_{i, k}+\phi_{w} w_{k} w_{i, k}\right] \phi_{w} } & +\frac{c_{\infty}^{2}}{\gamma} \phi_{0} \phi_{1} p_{c, i}= \\
= & -\phi_{0}\left(\rho_{s}+\phi_{1} \rho_{c}\right)\left[v_{i, t}+v_{k} v_{i, k}\right] \\
\phi_{0} \phi_{1}\left[\rho_{c, t}+v_{k} \rho_{c, k}+\phi_{w} w_{k} \rho_{c, k}\right]+\left[1+\phi_{0}\left(\rho_{s}+\phi_{1} \rho_{c}\right)\right] w_{k, k} \phi_{w} & =-\phi_{0}\left[\rho_{s, t}+v_{k} \rho_{s, k}\right] \\
\phi_{o} \phi_{1}\left(p_{c}-\gamma \rho_{c}\right) & =\frac{1}{2} \gamma(\gamma-1) \phi_{0}^{2}\left(\rho_{s}+\phi_{1} \rho_{c}\right)^{2} .
\end{aligned}
$$

It is important to note that the variable time is dimensional; length has been rescaled with $\ell$ so that the convective operators have dimensions of inverse time. The scalings for length and time will determine $\phi_{1}$ and $\phi_{w}$. The method of dominant balance, after choosing the acoustic length and time scales, indicates that the only self-consistent choices for the scale functions are $\phi_{w}=M_{t}^{2}$ and $\phi_{1}=M_{t}$. Additional details relevant to a similar procedure, though in a different context, can be found in Ristorcelli (1997A).

The compressible problem evolves on two time scales. The fast time scales with $\ell / c$. The slow time scales with $\ell / u_{c}$. Following the usual multi-scale ansatz the original time variable is replaced with two independent time variables, $[t] \Rightarrow\left[t_{0}, t_{1}\right] ; t_{0}$ is the fast time scale and $t_{1}$ is the slow time scale. The multi-time-scale ansat $z$ for a dependent variable is $f(x, t)=f\left(x, t_{0}, t_{1}\right)$. The time derivative, following a fluid particle of $f(x, t)$ is then written

$$
\frac{D}{D t} f(x, t)=\frac{D}{D t_{0}} f\left(x, t_{0}, t_{1}\right)+\epsilon \frac{D}{D t_{1}} f\left(x, t_{0}, t_{1}\right),
$$

where the small parameter is now understood to be $\epsilon=M_{t}$. The equations are made nondimensional with the integral length scale, $\ell$, and the fast time scale, $\ell / c$. The compressible dependent variables are now expanded in a regular perturbation expansion, $f=f_{0}+\epsilon f_{1}+\ldots$. A Reynolds decomposition of the velocity field is used: the mean flow is characterized by a shear of magnitude $S$. The mean flow is assumed, for present purposes, to be homogeneous with $V_{i, i}=0$. 
3.2. The leading order primitive equations. The leading order compressible problem satisfies the following momentum and continuity equations:

$$
\begin{aligned}
\frac{D}{D t_{0}} w_{0 i}+\left[\frac{S \ell}{c}\right] w_{0 k} V_{i, k}+p_{0, i} & =-p_{s}\left[\frac{S \ell}{c}\right] v_{k} V_{i, k} \\
\frac{D}{D t_{0}} p_{0}+w_{0 k, k} & =-\left[\frac{D}{D t_{1}} p_{s}+v_{k} p_{s, k}\right] .
\end{aligned}
$$

The leading order equation of state, $\gamma p_{0}=\rho_{0}$ has been used to eliminate the density in favor of pressure. There are several items worth noting:

1. The so-called gradient Mach number $\frac{S \ell}{c}$ appears as an independent parameter. It appears in what is called a production term (also sometimes called a refraction term).

2. The forcing terms are all functions of the slow time: $p_{s}=p_{s}\left(x, t_{1}\right), v_{i}=v_{i}\left(x, t_{1}\right)$.

3. The equations are linear: there is no nonlinear compressible-compressible mode coupling in the low Mach number limit. Nonlinearity is only a characteristic, as one might expect in a perturbative treatment, of the incompressible modes.

4. The solution is written as a sum of homogeneous and particular solutions which, to leading order, have a different dependence on the fast $\left(t_{0}\right)$ and slow $\left(t_{1}\right)$ time variables, Ristorcelli (1997A):

$$
\begin{aligned}
w_{0 i} & =w_{0 i E}\left(x, t_{0}\right)+w_{0 i P}\left(x, t_{1}\right) \\
p_{0} & =p_{0 E}\left(x, t_{0}\right)+p_{0 P}\left(x, t_{1}\right) .
\end{aligned}
$$

5. The initial value problem, $\left[w_{0 i E}\left(x, t_{0}\right), p_{0 E}\left(x, t_{0}\right)\right]$, is the solution of the homogeneous problem:

$$
\begin{aligned}
\frac{D}{D t_{0}} w_{0 i}+\left[\frac{S \ell}{c}\right] w_{0 k} V_{i, k}+p_{0, i} & =0 \\
\frac{D}{D t_{0}} p_{0}+w_{0 k, k} & =0 .
\end{aligned}
$$

These are the equations of an evanescent wave field. The solution is a function of, to leading order, of only the fast time.

3.3. A concomitant wave equation. If one takes the divergence of the momentum equation, (28), and the Lagrangian derivative of the continuity equation, (29), one obtains the following third-order wave equation with source:

$$
\frac{D}{D t_{0}}\left[\frac{D^{2}}{D t_{0}^{2}} p_{0}-\nabla^{2} p_{0}\right]+2\left[\frac{S \ell}{c}\right] V_{i, k} p_{0, i k}-4\left[\frac{S \ell}{c}\right]^{2} V_{k, i} V_{i, j} w_{0 j, k}=2\left[\frac{S \ell}{c}\right] V_{j, i} f_{i, j}
$$

On the right hand side $f_{i}=-p_{s}\left[\frac{S \ell}{c}\right] v_{k} V_{i, k}$, which is known in terms the solenoidal field. The problem can be viewed as a linear fourth order forced oscillator, whose natural time scale, $t_{0}$, is fast compared to the forcing time scale, $t_{1}$. The homogeneous solution satisfies the initial value problem and only varies on the fast time scale; the particular solution varies on the slow time scale. If the initial conditions are homogeneous, the evanescent wave field with the fast eigenmodes is not stimulated. In such a case the solution for the compressible modes follows the slow time forcing with a phase lag related to the damping.

In the absence of forcing the homogeneous problem satisfies the following wave equation

$$
\frac{D}{D t_{0}}\left[\frac{D^{2}}{D t_{0}^{2}} p_{0}-\nabla^{2} p_{0}\right]+2\left[\frac{S \ell}{c}\right] V_{i, k} p_{0, i k}=4\left[\frac{S \ell}{c}\right]^{2} V_{k, i} V_{i, j} w_{0 j, k}
$$

For a parallel unidirectional shear the right hand side is zero,

$$
\frac{D}{D t_{0}}\left[\frac{D^{2}}{D t_{0}^{2}} p_{0}-\nabla^{2} p_{0}\right]+2\left[\frac{S \ell}{c}\right] V_{i, k} p_{0, i k}=0
$$


and the order of the system is reduced. A equation similar in form has been deduced by Lilley (1996). Apart from some numerical factors the major difference from Lilley's result is that, as the equation is expressed on the fast time scale, it describes specifically the fast modes of the flow. This is the wave equations in a moving medium with refraction due to the mean shear. The mean shear alters the magnitude and direction of the wave field.

For a homogeneous medium this equation describes an evanescent wave field determined by the initial conditions. If the initial conditions are zero the solution remains zero; arbitrary non-zero initial conditions will stimulate the eigenmodes of this equation. These are waves with phase speed equal to speed of sound (unity in the present equations). The production (refraction) mechanism can amplify these modes. The production mechanism consequently leads to secular behavior; the growing amplified modes, extracting energy from the mean shear, will grow on a time scale $t_{0}$ such that the oscillations will no longer be small on a scale of the turbulent Mach number. (The above equations will also no longer be valid). This refraction mechanism is likely to be the source of the very large compressible pressure fluctuations seen in the compressible DNS, Blaisdell et al. (1991), Sarkar (1992). This possibility is the topic of research now in progress. Blaisdell et al. (1993) have also reported on a tilting of dilatational surfaces as the DNS proceeds; a refraction mechanism as indicated above would explain this behavior.

3.4. The concomitant vorticity equation. If one takes the curl of the momentum equation one obtains the vorticity equation:

$$
\frac{D}{D t_{0}} \omega_{0 q}+\left[\frac{S \ell}{c}\right] \varepsilon_{q i j}\left[w_{0 k}, j V_{i, k}+w_{0 i, k} V_{k}, j\right]=-\left[\frac{S \ell}{c}\right] \varepsilon_{q i j}\left(p_{s} v_{k}\right),{ }_{j} V_{i, k}
$$

The compressible fast modes are not irrotational (even if they start irrotational). It appears that it is inappropriate to call the field acoustic: there are rotational modes that propagate with acoustic phase speed which are stimulated by the initial conditions. Like the leading order compressible pressure field the compressible vortical modes can be amplified by extracting energy from the mean shear.

3.5. Summary of two-timing results. A simple two-time analysis has indicated some very interesting and simple models for the compressible portions of a turbulent flow. The fast compressible modes are governed by a forced convective wave equation with production (refraction) terms; this can be understood as a linear forced oscillator model for compressible effects. In a medium undergoing a simple homogeneous shear the following linear wave equation describing the evolution of the initial conditions determining an evanescent compressible wave field with acoustic phase speed:

$$
\frac{D}{D t_{0}}\left[\frac{D^{2}}{D t_{0}^{2}} p_{0}-\nabla^{2} p_{0}\right]+2\left[\frac{S \ell}{c}\right] V_{i, k} p_{0, i k}=0 .
$$

This is essentially a third order linear oscillator, modulo mean convection effects. The equation contains the production (refraction) term, $V_{i, k} p_{0, i k}$, which will produce secular growth that is a possible mechanism for the large oscillations seen in the compressible DNS in the presence of shear.

4. Compressible turbulence modeling. An investigation, using recent DNS data, Blaisdell (1996), of the scalings obtained for the pressure-dilatation and the dilatational dissipation by Ristorcelli (1997) is the primary subject of this section. The representations given in Ristorcelli (1997) were obtained using simple scaling arguments regarding the effects of compressibility, a singular perturbation idea and the methods of statistical fluid mechanics. While the results are expressed in the context of a statistical turbulence closure, they provide, with few phenomenological assumptions, an interesting and clear physical model for the scalar 
effects of compressibility. The Ristorcelli (1997) analysis is relevant to shear flows with negligible bulk dilatation and small $M_{t}^{2}$. These restrictions are also met in a wide number of engineering flows ranging from simple shear layers of fundamental and practical interest, Papamoschou and Roshko (1988), as well as the complex shear layers associated with supersonic mixing enhancement, Gutmark et al. (1995). In most of these supersonic shear layers a Mach number based on the fluctuating velocity of the fluid particle is small. For example, in a Mach 4 mean flow with a turbulence intensity of 8 per cent has a turbulent Mach number of $M_{t}=0.32$. The square of this turbulent Mach number, the appropriate perturbation expansion parameter arising from the Navier-Stokes equations, $M_{t}^{2} \sim 0.1$, is small. These restrictions are also met in the DNS of homogeneous shear; the homogeneous shear is our laboratory experiment.

4.0.1. A pseudo-sound analysis for the effects of compressibility. The existence of a small parameter allows some analytical results. In Ristorcelli (1997) a low turbulent Mach number expansion for the compressible Navier Stokes equations produced a diagnostic relationship for the dilatation. To leading order, the density fluctuations are given, in nondimensional units, by the solenoidal pressure fluctuations, $\gamma \rho_{s}=p_{s}$ and the continuity equation becomes a diagnostic relation for the fluctuating dilatation,

$$
-\gamma d=p_{s, t}+v_{k} p_{s, k}
$$

One does not need to obtain a solution to the evolution equation for the compressible velocity field, $w_{i}$, in order to obtain, to leading order, its dilatation, $d=w_{i, i}$. This is a useful result. The dilatation is determined by local fluctuations of the pressure and velocity; it is the rate of change of the solenoidal pressure field $p_{s, j j}=\left(v_{i} v_{j}\right)_{, i j}$, following a fluid particle.

In a two equation turbulence closure an equation for the kinetic energy of the fluctuations, $k$, is carried. In the kinetic energy equation two new covariances with the fluctuating dilatation appear: the pressuredilatation and the dilatational dissipation. Constitutive relations for the pressure-dilatation and the dilatational dissipation can be found by taking the appropriate moments of the diagnostic relation for the dilatation to produce, to leading order in $M_{t}^{2}$,

$$
\begin{aligned}
-2 \gamma\langle p d\rangle & =\frac{D}{D t}\left\langle p_{s} p_{s}\right\rangle \\
\gamma^{2}\langle d d\rangle & =\left\langle\dot{p_{s}} \dot{p_{s}}\right\rangle+2\left\langle\dot{p_{s}} v_{k} p_{s, k}\right\rangle+\left\langle v_{k} p_{s, k} v_{q} p_{s, q}\right\rangle
\end{aligned}
$$

The substituting of the solutions to $p_{s}, j_{j}=\left(v_{i} v_{j}\right)_{i j}$, into the right hand side of the above constitutive relations has been used to obtain representations for $\langle p d\rangle$ and $\varepsilon_{c}$, Ristorcelli (1997).

4.1. The dilatational dissipation. The local dilatational dissipation is comprised of a slow and a rapid part: $\varepsilon_{c}=\varepsilon_{c}^{r}+\varepsilon_{c}^{s}$, where

$$
\begin{aligned}
\varepsilon_{\mathrm{c}}^{s} & =\frac{16}{3 \alpha^{2}} \frac{M_{t}^{4}}{R_{t}} \varepsilon_{s}\left[I_{2}^{s}+6 I_{1}^{s} I_{3}^{s}\right] \\
\varepsilon_{\mathrm{c}}^{r} & =\left(\frac{2}{3}\right)^{5} \frac{M_{t}^{4} \hat{S}^{2}}{R_{t}} \varepsilon_{s}\left[3+5 R^{2}\right]\left[\frac{3}{5} I_{3}^{r}+\left(\frac{\hat{S}}{15}\right)^{2}\left[13+15 R^{2}\right] \alpha^{2} I_{1}^{r}\right]
\end{aligned}
$$

Here $R^{2}=W^{2} / S^{2}$ is the mean rotation to strain ratio; $R=1$ for a pure shear. The nondimensional strain and rotation rates are given by: $\hat{S}^{2}=\left(S k / \varepsilon_{s}\right)^{2}, \hat{W}^{2}=\left(W k / \varepsilon_{s}\right)^{2}$ where $S=\sqrt{S_{i j} S_{i j}}$ and $W=$ $\sqrt{W_{i j} W_{i j}}$. The strain and rotation tensors are defined in analogy with the incompressible casc, i.e. traceless, $S_{i j}=\frac{1}{2}\left[V_{i}, j+V_{j, i}-\frac{2}{3} D \delta_{i j}\right], W_{i j}=\frac{1}{2}\left[V_{i},{ }_{j}-V_{j}, i\right] . \quad M_{t}$ is the turbulent Mach number, $M_{t}^{2}=\frac{2}{3} k / c^{2}$, where $c^{2}=\gamma\langle p\rangle /\langle\rho\rangle$ is the local sound speed. The traditional definition of the Mach number is used, 
$M_{t}=u_{c} / c, u_{c}^{2}=\frac{2}{3} k$. The turbulent Reynolds number is given by $R_{t}=\frac{u_{c} \ell}{\nu}=\frac{4 k^{2}}{9 \varepsilon \nu}$ using the well known Kolmogorov scaling $\varepsilon_{s}=\alpha u_{c}^{3} / \ell$ with $\alpha \approx 1$.

The constants, denoted by $I_{i}$, in these expressions are not adjustable empirical calibration constant obtained by matching calculations to obtain the trends in experimental data; they are fully specified and measurable quantities. They are defined in terms of diverse integrals of the longitudinal correlation. The reader is referred to Ristorcelli (1997) for additional details.

The behavior that is most easily explored - in as much as the isotropic decay is the cheapest of DNS to conduct - is the scaling with turbulent Mach number. The dilatational dissipation scales as

$$
\varepsilon_{c}^{s} \sim M_{t}^{4} \varepsilon_{s} .
$$

Figure 2 used in the discussion on initial conditions in $\S 2$ is relevant. With the "consistent" initial conditions derived from finite Mach number asymptotics, a $M_{t}^{4}$ scaling is clearly seen. A similar $M_{t}^{4}$ scaling has been seen in the EDQNM simulations of the Bertoglio group at Lyon. The Reynolds number dependence is not so easily explored as, in DNS, the Reynolds number cannot be varied over a substantial range. In the EDQMN simulations of the Bertoglio group a dependence on the Reynolds number is seen.

4.2. The pressure-dilatation. In the asymptotic analysis of Ristorcelli (1997) the following representation for the pressure-dilatation was, to leading order, obtained:

$$
\left.<p d>=-\chi_{p d} M_{t}^{2}\left[\bar{\rho} P_{k}-\bar{\rho} \varepsilon+T_{k}\right]\left(P_{T}+\bar{\rho} \varepsilon+T_{T}\right)\right]-\bar{\rho} k M_{t}^{2} \chi_{p d}^{\tau} \frac{D}{D t} \mathcal{T}
$$

where

$$
\begin{aligned}
\chi_{p d} & =\frac{2 I_{p d}}{1+2 I_{p d} M_{t}^{2}+\frac{3}{2} I_{p d} M_{t}^{4} \gamma(\gamma-1)} \\
\chi_{p d}^{r} & =\frac{I_{p d}^{r}}{1+2 I_{p d} M_{t}^{2}+\frac{3}{2} I_{p d} M_{t}^{4} \gamma(\gamma-1)} \\
I_{p d} & =\frac{2}{3} I_{1}^{s}+I_{p d}^{r}\left[3 \hat{S}^{2}+5 \hat{W}^{2}\right] \\
I_{p d}^{r} & =\frac{1}{30}\left(\frac{2}{3}\right)^{3} \alpha^{2} I_{1}^{r} .
\end{aligned}
$$

Here $T=\left[3 \hat{S}^{2}+5 \hat{W}^{2}\right]$. The constants, denoted by $I_{i}$, in these expressions are fully specified by the analysis. For the present article, concerned as it is with the scalings of the compressible covariances, their exact values are not relevant.

4.2.1. Pressure-dilatation in the isotropic decay. For the isotropic decay the expression for the pressure-dilatation becomes

$$
<p d>=\chi_{p d} M_{t}^{2} \varepsilon_{s} .
$$

Here $\bar{\rho}$ has been set to unity. The sign of $\langle p d\rangle$ is positive indicating a net transfer of energy from potential to kinetic modes. Rearranging, to isolate the scaling, produces

$$
\frac{<p d>}{M_{t}^{2} \varepsilon_{s}}=\chi_{p d}=\frac{\frac{4}{3} I_{1}^{s}}{1+\frac{4}{3} I_{1}^{s} M_{t}^{2}}
$$

Terms of order $M_{t}^{4}$ have been dropped. Earlier estimates given in Ristorcelli (1995), shown above, indicate $I_{1}^{s}=0.5-0.3$. The theory therefore predicts an asymptote for $\chi_{p d}$ as the turbulent Mach number vanishes:

$$
\chi_{p d} \rightarrow 0.666-0.40 \quad \text { as } \quad M_{t}^{2} \rightarrow 0 .
$$


The DNS results, shown in Figure 4, were provided by Blaisdell for three different initial turbulent Mach numbers. As a service to the reader, the figure identifies two definitions of the turbulent Mach number: that used by Blaisdell et al. (1993) in their simulations, $M_{t}^{b}$, and that which comes from the traditional definition of the Mach number. The present compressible DNS reflect a consistent set of initial conditions as described above and in Ristorcelli and Blaisdell (1997).

The agreement with the DNS, shown in Figure 4, is very good. The analysis is consistent with the DNS without a posteriori adjustment of constants. The actual values of the constant could in principle be calculated from the DNS. As they are expected to be weakly dependent on initial conditions, this course is not followed further - what has been presented is sufficient for verification. Moreover, the slow portion of the pressure-dilatation is nominal compared to the rapid portion which is the most important contributor in the shear flows of interest.

4.2.2. Homogeneous shear. The pressure-dilatation in the homogeneous shear is now investigated. The instantaneous pressure-dilatation is seen in Figure 5. Also shown are its averaged values following the procedure of Sarkar (1992). Here the time integral of the pressure-dilatation has been taken: the vertical axis being $-\frac{1}{S t} \int \frac{\langle p d\rangle}{\varepsilon_{s}} d(S t)$. The oscillations in the pressure-dilatation associated with the relaxation from initial conditions are not seen. There is, nonetheless, a build up of the oscillations which has been linked to the compressible component of the pressure field, Sarkar (1992), Blaisdell and Sarkar (1993). A plausible physical mechanism for this behavior was given in $\S 3$.

For homogeneous shear, the expression for the pressure-dilatation can be simplified. For simple shear $\mathcal{T}=8 \hat{S}^{2}$ one obtains

$$
<p d>=-\chi_{p d} M_{t}^{2}\left[P_{k}-\varepsilon_{s}\right]-k M_{t}^{2} \chi_{p d}^{\tau} 8 \frac{D}{D t} \hat{S}^{2}
$$

Here $\hat{S}=\frac{S k}{\varepsilon_{a}}$. For Blaisdell's homogeneous shear, in which $S=$ const., the expression can be rearranged

$$
<p d>=-\chi_{p d} M_{t}^{2} \varepsilon_{s}\left[\frac{P_{k}}{\varepsilon_{s}}-1\right]-16 \chi_{p d}^{r} M_{t}^{2} \varepsilon_{s}\left(\frac{S k}{\varepsilon_{s}}\right)^{2} \frac{D}{D(S t)} \frac{S k}{\varepsilon_{s}} .
$$

Note that the cocfficient of the first term, $\chi_{p d}$, ignoring the small slow pressure contribution, scales as $\chi_{p d} \sim\left(\frac{S k}{\varepsilon_{s}}\right)^{2}$; thus accounting for the definitions of the $\chi^{\prime}$ s, the pressure-dilatation can be rewritten as

$$
<p d>\sim-\left[\alpha^{2}\left(\frac{S k}{\varepsilon_{s}}\right)^{2} M_{t}^{2} \varepsilon_{s}\left(\frac{P_{k}}{\varepsilon_{s}}-1\right)\right] I_{1}^{r}\left[1+\frac{1}{\frac{P_{k}}{\varepsilon_{s}}-1} \frac{D}{D(S t)} \frac{S k}{\varepsilon_{s}}\right] .
$$

As the flow evolves, it is expected that $I_{1}^{r} \rightarrow$ const and $\frac{D}{D(S t)} \frac{S k}{\varepsilon_{s}} \rightarrow 0$ thus $\left[1+\frac{1}{\frac{p_{k}}{\varepsilon_{s}}-1} \frac{D}{D(S t)} \frac{S k}{\varepsilon_{s}}\right] \rightarrow 1$. The scaling of $\langle p d\rangle$ with the term in the first set of brackets will be investigated.

4.2.3. Pressure-dilatation scalings. The appropriately scaled integrals of $\langle p d\rangle$ will now be taken. In this way one can establish whether the scalings predicted by the model are useful. In the latter portions of Blaisdell's DNS, $S t>10$, about three to four eddy turnovers past initialization, a structural equilibrium is approached. In this region the scaling, given by the analysis above, indicates

$$
<p d>\sim-\left[\alpha^{2}\left(\frac{S k}{\varepsilon_{s}}\right)^{2} M_{t}^{2} \varepsilon_{s}\left(\frac{P_{k}}{\varepsilon_{s}}-1\right)\right] I_{1}^{r}
$$

is investigated. The time integral $\frac{1}{S t-S t_{0}} \int() d(S t) \equiv \int_{S T}$ of diverse normalizations of will be taken. The integrals

$$
I_{0}=\int_{S T}<p d>
$$




$$
\begin{aligned}
I_{1} & =\int_{S T} \frac{\langle p d\rangle}{\epsilon_{s}} \\
I_{2} & =\int_{S T} \frac{<p d\rangle}{\varepsilon_{s}\left[\frac{P_{k}}{\varepsilon}-1\right]} \\
I_{3} & =\int_{S T} \frac{<p d>}{M_{t}^{2} \varepsilon_{s}\left[\frac{P_{k}}{\varepsilon}-1\right]\left(\frac{S k}{\varepsilon}\right)^{2}} \\
I_{4} & =\int_{S T} \frac{<p d>}{\alpha^{2} M_{t}^{2} \varepsilon_{s}\left[\frac{P_{k}}{\varepsilon}-1\right]\left(\frac{S k}{\varepsilon}\right)^{2}}
\end{aligned}
$$

will be computed from the data. The integrals are shown in Figure 5. The integration starts at $S t=9$; the integrals are normalized by their values at $S t=10$. The integration is started at $S t>9$ so that nonequilibrium effects associated with the development of the turbulence from the initial conditions has faded. During such transient periods integrals in the pressure-dilatation model will not be constant. For $S t>9$ the quantity $\frac{1}{S t} \int I_{1}^{r} d(S t) \sim I_{1}^{r}$ and $\frac{D}{D t} \hat{S} \rightarrow 0$ and the validity of the scaling can be satisfactorily assessed. If the scaling suggested by the analysis of Ristorcelli $(1995,1997)$ is correct the last integral, $I_{4}$, will be approximately constant once an average is performed over a few of the oscillations. The period of time $10<S t<16$ corresponds to about one eddy turnover time.

4.2.4. The gradient Mach number. The largest relative collapse of the normalized $\langle p d\rangle$ integrals is the collapse from $I_{2}$ to $I_{3}$. The $I_{3}$ includes the quantities $M_{t}^{2}\left(\frac{S k}{\varepsilon}\right)^{2}$. The quantity $M_{t}\left(\frac{S k}{\varepsilon}\right)$ can be understood as a gradient Mach number. The pressure-dilatation is a strong function of the gradient Mach number. Sarkar (1995) has defined a gradient Mach number as $M_{g}=S \ell / c$; the transverse two-point correlation of the longitudinal velocity is used as the length scale, $\ell$. In this article $\ell$ will be taken as the traditionally defined longitudinal length scale that occurs in the Kolmogorov scaling: $\ell=\alpha(2 k / 3)^{3 / 2} / \varepsilon_{s}$. In which case a mean strain Mach number is defined:

$$
M_{S}=\frac{S \ell}{c}=\alpha \frac{2}{3} \frac{S k}{\varepsilon_{s}} M_{t}=\alpha \frac{2}{3} \hat{S} M_{t} \simeq \frac{2}{3} \hat{S} M_{t} .
$$

The approximation $\alpha \approx 1$ has been made. In fact, the curve $I_{3}$ overshoots the optimum collapse (a horizontal line): the gradient Mach number is increasing faster than $\langle p d\rangle$. The curve, $I_{4}$, which accounts for the Kolmogorov coefficient in addition to the scalings of $I_{3}$, is a further improvement.

4.2.5. The Kolmogorov scaling coefficient. A new feature associated with compressibility for this class of turbulence closures, is the appearance of the Kolmogorov scaling coefficient, $\alpha . M_{t}$ and $\frac{S k}{\epsilon_{s}}$ are not new quantities for describing turbulence in single point closures; $\alpha$, however, is new. The quantities $M_{t}$ and $\frac{S k}{\varepsilon_{s}}$ are computed as part of a turbulence model simulation; $\alpha$ however is not. This distinction is made in order to recognize $\alpha$ as a new independent quantity. The value of $\alpha$ can be thought of as describing large scale structural aspects of the flow: after all, it relates the kinetic energy, its cascade rate, and the two-point correlations.

The Kolmogorov parameter $\alpha$ appears because it is has been necessary to evaluate spatial two-point integrals that were made nondimensional using $\ell$, Ristorcelli (1997). The dependence on the Kolmogorov scaling coefficient appears when the integral length scale, $\ell$, is eliminated in favour of $k$ and $\varepsilon$ which are carried in standard turbulence closures. Such a procedure does not appear in developments for incompressible closures - the incompressible pressure-strain is independent of length scale.

The values used for $\alpha$ come from Blaisdell's DNS: the longitudinal integral length scale, $\ell, k$ and $\varepsilon_{s}$ are calculated from the DNS and then the Kolmogorov relationship, $\varepsilon=\alpha\left(\frac{2}{3} k\right)^{3 / 2} / \ell$ is used to find $\alpha$. 
The Kolmogorov constant is thought to be a universal constant for high Reynolds number isotropic turbulence; for nonideal finite Reynolds number anisotropic turbulence it is a flow specific quantity. It is this fact that makes creating a singel point turbulence model difficult; a choice for $\alpha$ must be made and for any given flow the choice is not, a priori, known.

4.3. Comparison with select pressure-dilatation models. The analysis of Ristorcelli (1997) has treated the case in which dilatational fuctuations come from the turbulence and not an cvanescent wave field due to initial conditions. A good clean set of DNS calculations, uncontaminated by arbitrary initial conditions, now exists for the homogeneous shear. Blaisdell's new calculations allow a study of the effects of compressibility due to the non-zero Mach number of the vortical fluctuations. This is the simplest flow possible and such dilatational fluctuations will be directly related to turbulence present in enginecring flows of interest. Comparison with published work on the pressure-dilatation covariance is now made.

Zeman's (1991) model is given by

$$
<p d>=-\frac{1}{2 \bar{\rho} c^{2}} \frac{D}{D t}<p p>=\frac{1}{2 \bar{\rho} c^{2}} \frac{<p p>-<p_{e} p_{e}>}{\tau_{a}},
$$

where $\tau_{a}=0.13(2 k / \varepsilon) M_{t}^{b}$. The variance $<p_{e} p_{e}>$, which represents an equilibrium level of pressure variance; it is given phenomenologically:

$$
<p_{e} p_{e}>=\frac{\alpha M_{t}^{2}+M_{t}^{4}}{1+\alpha M_{t}^{2}+M_{t}^{4}} .
$$

Zeman's modeling is not consistent with low $M_{t}^{2}$ asymptotics: as $M_{t}^{2} \rightarrow 0$ Zeman's phenomenology indicates $\langle p p\rangle \rightarrow\left\langle p_{e} p_{e}\right\rangle \rightarrow 0$. The pressure variance must, however, asymptote to the incompressible pressure variance, $\langle p p\rangle \rightarrow\left\langle p_{s} p_{s}\right\rangle$, which will be a function of the energy of the turbulence. Zeman (1991) apparently treats the model problem in which there are arbitrary pressure fluctuations due to initial conditions - the initial value problem. The compressible effects due to the compressible nature of the turbulence itself is missed. Over a time scale of the turbulence $\tau_{a} / M_{t}$, the pressure variance predicted by this model will have equilibrated to $\left\langle p_{e} p_{e}\right\rangle$ and the pressure-dilatation will be zero. This is not seen in the DNS of either the isotropic decay or the homogeneous shear. For this reason the Zeman modeling is not further investigated.

Aupoix, Blaisdell, Reynolds and Zeman (1990) have, apparently, a similar problem in mind; their resolution is, however, more general. Their value of the pressure-dilatation is obtained from an evolution equation:

$$
\frac{D}{D t}<p d>=-C_{1} \frac{M_{t}^{2}}{\tau_{a}} \frac{D}{D t} k-\frac{C_{2}}{\tau_{a}}<p d>
$$

Again $\tau_{a}$ is a fast "acoustic" time scale. If one inserts the evolution equation for $k, \frac{D}{D t} k=P_{k}-\varepsilon+<p d>$ one obtains:

$$
\frac{D}{D t}<p d>=-\left[\left(C_{1} M_{t}^{2}+C_{2}\right)<p d>+C_{1} M_{t}^{2}\left(P_{k}-\varepsilon_{s}\right)\right] / \tau_{a}
$$

The density has been set to unity. On the slow manifold, characteristic of the decay, the fixed point solution yields

$$
<p d>=-\frac{C_{1} M_{t}^{2}}{C_{2}+C_{1} M_{t}^{2}}\left[P_{k}-\varepsilon_{s}\right]
$$

which has a form similar to the present model. 
Sarkar's (1992) pressure-dilatation model for flows without bulk dilatation, is given by

$$
<p d>=-0.15 M_{t}^{2}\left[\frac{P_{k}}{M_{t}}-\frac{4}{3} \varepsilon_{s}\right]
$$

This is the same form of the model as used in the simulations of Abid (1994). In case of misunderstanding it should be pointed out that there are no singularities at low turbulent Mach number in Sarkar's (1992) model.

4.3.1. Model comparison - the isotropic decay. For the isotropic decay the Aupoix et al. (1990) model, the Sarkar (1992) model and the present model given above are all, in the low $M_{t}$ limit, topologically similar. The three models are, respectively,

$$
\begin{aligned}
& <p d>=\frac{C_{1} M_{t}^{2}}{C_{2}+C_{1} M_{t}^{2}} \varepsilon \\
& <p d>=0.2 M_{t}^{2} \varepsilon \\
& <p d>=\chi_{p d} M_{t}^{2} \varepsilon_{s} .
\end{aligned}
$$

The coefficients for Sarkar and Aupoix are determined empirically by matching with compressible simulations; for the present model the coefficent, $\chi_{p d}$, is related to a two-point integral and is obtained in a way not related to any compressible experiments. The coefficients in the models show $\frac{\langle p d\rangle}{M_{t}^{2} \varepsilon} \sim 1.25,0.2$ and .6 for the Aupoix, Sarkar and Ristorcelli models. The DNS of Blaisdell, Figure 2, indicates a value $\approx 0.6$ is the better value for the coefficient.

4.3.2. Model comparison - the homogeneous shear. For the isotropic decay the Aupoix et al. (1990) model, the Sarkar (1992) model and the present model are, in the low $M_{t}$ limit, topologically similar. For the homogeneous shear differences between the models become apparent.

$$
\begin{aligned}
& <p d>=-\frac{C_{1} M_{t}^{2}}{C_{2}+C_{1} M_{t}^{2}} \varepsilon_{s}\left[\frac{P_{k}}{\varepsilon_{s}}-1\right] \\
& <p d>=-0.15 M_{t}^{2} \varepsilon_{s}\left[\frac{P_{k}}{\varepsilon_{s} M_{t}}-\frac{4}{3}\right] \\
& <p d>=-\chi_{p d} M_{t}^{2} \varepsilon_{s}\left[\frac{P_{k}}{\varepsilon_{s}}-1\right] .
\end{aligned}
$$

The models have been arranged to display some of their similar features; certain groupings of parameters have been emphasized. In case of misunderstanding it should be pointed out that there are no singularities at low turbulent Mach number in Sarkar's (1992) model. The grouping in the models highlights an $M_{t}^{2}$ dependence; the term in the brackets can be understood as representing a (weighted) departure from equilibrium. Note that all three models allow for a change in sign of $\langle p d\rangle$. For the Sarkar model the change in sign is dependent on the turbulent Mach number. For Aupoix and Ristorcelli models the change in sign is simply dependent on the whether production exceeds dissipation.

The present model, (63), features an additional dependence on the Kolmogorov scaling parameter, $\alpha$, and the relative strain $\frac{S k}{\varepsilon_{s}}$. This occurs through the definition of $\chi_{p d}$; recall that $\chi_{p d} \sim \alpha^{2}\left(\frac{S k}{\varepsilon_{s}}\right)^{2}$. Fürthermore, the combination $M_{t} \frac{S k}{\varepsilon_{s}}$ can be understood as a gradient Mach number; the combination $\chi_{p d} M_{t}^{2}$ indicates a proportionality of the pressure-dilatation to the square of the gradient Mach number.

A comparison of the different models with DNS is indicated in Figure 6. The simulation includes a considerable "non-equilibrium" portion of the DNS. The nonconstant values of the integrals $I_{i}$ have been included in the present form of the model: the values of the required $I_{i}$ are calculated from the DNS from 
their definition. At present we are primarily interested in the scalings - all curves have been normalized by their value at the end of the simulation.

Zeman's modeling, Zeman and Coleman (1991), is also not appropriate for the homogeneous shear with consistent initial conditions for the reasons given above. Durbin and Zeman (1992) have made several improvements to Zeman's original ideas - the vortical fluctuations are recognized as producing dilatational fluctuations, and thus Durbin and Zeman (1992) is consistent with low Mach number asymptotics. Their closure is more complex; requiring, in addition to the solution an evolution equation the pressure variance, but also an equation for a length scale associated with the pressure field. Their development treats rapidly distorted flows and their modelling and validation focusses on flows with large mean dilatations - a flow in which the $\langle p d\rangle$ is important. The present development is focussed on compressible shear flows and a first step in the development of a compressible closure that might explain and predict the growth rate suppression seen in many mixing layer experiments. The mixing layer is not a case of a rapidly distorted flow.

4.4. Turbulence modeling conclusions. A simple low Mach number analysis of the Navier Stokes equations has related the fluctuating dilatation to the solenoidal pressure field, Ristorcelli (1997). This is used to produce constitutive relations for the pressure-dilatation and the dilatational dissipation. The scalings of the these expressions with turbulent Mach number, relative strain rate, gradient Mach number and Kolomogorov scaling coefficient have been shown to collapse the DNS data.

The DNS - with consistent initial conditions - indicates that the dilatational dissipation scales as $M_{t}^{4}$. As such, the dilatational dissipation does not appear to be an item of any concern in low turbulent Mach number flows of interest to high speed (supersonic) aerodynamics. The dilatational dissipation with its $M_{t}^{4}$ dependence certainly cannot explain the reduced growth rate of the compressible mixing layer, Thangam et al. (1996a, 1996b). This is consistent with recent simulations, Sarkar (1995), Vreman et al. (1996), Simone et al. (1997).

The pressure-dilatation is found to be a nonequilibrium phenomenon. It scales as $\alpha^{2} M_{t}^{2}\left(\frac{S k}{\varepsilon_{a}}\right)^{2}\left[P_{k} / \varepsilon_{s}-1\right]$. For it to be important requires both 1) the square of the gradient Mach number, $M_{S}^{2}$, to be substantial and 2) for the flow to be out of equilibrium $P_{k} \neq \varepsilon$. The pressure-dilatation has been observed and is predicted to be either positive or negative. Its dependence on the non-equilibrium nature of the flow, $P_{k} \neq \varepsilon$, indicates that the pressure-dilatation can be either stabilizing or destabilizing. These trends appear consistent with the DNS of Simone et al. (1997), in which such a dependence on $P_{k} / \varepsilon$, as related to and implied by the evolution of the anisotropy, $b_{12}=\frac{\langle u v\rangle}{2 k}$, from isotropic initial conditions, can be inferred.

The homogeneous shear is arguably the most non-equilibrium flow of the the diverse benchmark flows. Accordingly the pressure-dilatation, for this class of flows, will be at its most important. In as much as many aerodynamic flows are close to equilibrium, it also appears that the pressure-dilatation with $\alpha \approx 1$ cannot explain the dramatically reduced growth rate of the mixing layer, Thangam et al. (1996a, 1996b). A value of $\alpha \approx 1$ is a reasonable guess for an equilibrium turbulence; an $\alpha \approx 1$ appears to account for about one fifth of the reduction of the growth rate. A choice of $\alpha \approx 2.5$ would produce the observed reduction of the mixing layer growth rate; this, however, would be no more accurate a representation of the physics than when the dilatation dissipation was argued to account for the reduction of the growth rate of the mixing layer.

The present discussion treats only the "scalar" effects of compressibility - the changes in the turbulence energy, $k$, due to the dilatational covariances in the energy budget. The dilatational covariances cannot account for the reduction in the shear anisotropy, $b_{12}$, or the normal anisotropy, $b_{11}$, so important in the production of the shear stress, $\left\langle v_{1} v_{2}\right\rangle$. To account for these more substantial structural effects appears to require a compressible pressure-strain representation accounting for the effects of compressibility in the 
evolution equations for the Reynold stresses.

In the pseudo-sound analysis the pressure-dilatation is found to be a function of the Kolmogorov scaling coefficient and this is anticipated to be an important (and potentially difficult) feature in single point closures for the effects of compressibility. The Kolmogorov coefficient, a reflection of an equilibrium cascade and an inertial subrange is, in general, a flow dependent quantity: there is little known about its dependence in non-ideal - anisotropic, strained, inhomogeneous - flow situations. The appearance of the Kolmogorov coefficient, in as much as it links the energy, the spectral flux, and a two-point length scale, is an indication of dependence on large scale structure.

5. Summary. This article has described the consequences of low Mach number expansions of the Navier Stokes equations for three different problems. In $§ 2$, time was spent developing physically relevant initial conditions for DNS of compressible turbulence. From Blaisdell's DNS it was shown that initial conditions can have a substantial effect on the scalings of the dilatational dissipation. It is clear that the DNS of compressible homogeneous turbulence - in whihe the medium can sustain a wave field in addition to the nonlinear advective field - needs careful consideration.

In $\S 3$, a two-timing perturbation method indicated that the fast compressible modes of a turbulent field are, to lowest order, described by a forced linear wave equation with refraction. Compressibility effects can be understood, as indicated by the two-time perturbation analysis, using a forced linear oscillator analog.

Several points relevant to understanding initial conditions for DNS of compressible turbulence stand out:

1. Inconsistent initial conditions stimulate modes with acoustic phase speed.

2. Consistent initial conditions eliminate the oscillations associated with the initial value problem.

3. Modes with acoustic phase speeds are solutions to the initial value problem and cannot be referred to as compressible turbulence which would have an advective phase speed.

4. Turbulence models for acoustic modes are as arbitrary as the initial conditions.

5. The initial value problem associated with inconsistent initial conditions which indicates an $M_{t}^{2}$ scaling for the dilatational dissipation. This is a scaling that is consistent with the presence of an evanescent wave field due to the initial conditions. The dilatational dissipation due to the compressible nature of the turbulence has an $M_{t}^{4}$ scaling.

A single time small Mach number analysis was used to produce models for the covariances with the dilatation. The analysis indicated the importance and role of several nondimensional parameters characterizing the compressibility of the turbulence. The nondimensional parameters that arise from a pseudo-sound analysis of weakly compressible turbulence are

1. The square of the turbulent Mach number: $M_{t}^{2}$.

2. The gradient Mach number: $M_{S}=\frac{2}{3} \frac{S k}{\varepsilon} M_{t}$.

3. The Kolmogorov scaling coefficient: $\alpha=\varepsilon_{s} \ell / u_{c}^{3}$.

4. The departure from equilibrium: $\frac{P_{k}}{\varepsilon_{s}}-1$.

Some of these parameters have been identified in previous works.

From the turbulence modeling point of view the results of the pseudo-sound analysis have indicated that: 1.) Compressible dissipation will not be an important phenomenon for low turbulent Mach numbers as occurs in many aerodynamic applications; 2.) The pressure-dilatation will only be important to the degree that the flow is out of equilibrium. It might also be mentioned that the pseudo-sound analysis predicts, contrary to expectations, a dilatational dissipation that depends on the Reynolds number. This is a precautionary statement: it is as example of a situation in which extrapolating a model for the effects of turbulence based 
on DNS data would be inappropriate for high Reynolds number flows. Presently DNS can not explore a wide enough Reynolds number range and, as a consequence, Reynolds number dependent phenomena can potentially be missed.

The low Mach number pscudo-sound analysis, in as much as it is supported by the DNS, appears to be a useful tool: it appears to have successfully

- Eliminated the fast modes associated with the initial value problem.

- Predicted the $M_{t}^{4}$ scalings of the dilatational dissipation and its consequent neglectability.

- Predicted the scalings of the pressure-dilatation.

With the successes of such a rational procedure incorporating the more complex effects of compressibility can be done with more confidence. Of particular interest is the very difficult problem of the pressure-strain covariance. The importance the pressure-strain has been indicated in Blaisdell and Sarkar (1992), Vreman et al. (1996), Sarkar (1995), Simone et al. (1997), Freund et al. (1997).

\section{Acknowledgements}

A substantial debt is owed to Professor G. Blaisdell for providing the results of his new DNS calculations with "consistent initial conditions."

\section{REFERENCES}

B. Aupoix, G.A. Blaisdell, W.C. Reynolds, O. Zeman (1990), Modeling the turbulent kinetic energy equation for compressible turbulence. Proceedings of the Summer Program, Center for Turbulence Research, Stanford U., Stanford, CA.

G.A. Blaisdell (1996), Personal communication.

G.A. Blaisdell, N.N. Mansour, W.C. Reynolds (1991), Compressibility effects on the growth and structure of homogeneous turbulent shear flow. Report TF-50, Department of Mechanical Engineering, Stanford University, Stanford, CA.

G.A. Blaisdell, N.N. MANSOUR AND W.C. ReYnolds (1993), Compressibility effects on the growth and structure of homogeneous turbulent shear flow. J. Fluid Mech. 256, p. 443.

G.A. BLAISDELL AND S.S. SARKAR (1993), Investigation of the pressure-strain correlation in compressible homogeneous turbulent shear flow. ASME FED-vol 151, Transitional and Turbulent Compressible Flows.

P. Bradshaw (1977), Compressible turbulent shear layers. Ann. Rev. Fluid Mech. 9:33.

J.B. Freund, S.K. Lele, P. MoIN (1997), Direct simulation of a supersonic round turbulent shear layer. AIAA-97-0760. 35th Aerospaces Sciences Meeting and Exhibit, Reno, NV

J.B. Freund, P. MOIN, S.K. Lele, (1997), Compressibility effects in a turbulent annular mixing layer. 1997 Report TF-72, Stanford University, Mechanical Engineering.

E.J. GutMARK, K.C. Schadow AND K.H. YU (1995), Mixing enhancement in supersonic free shear layers. Ann. Rev. Fluid Mech. 27, p. 375.

D. Papamoschou AND A. Roshko (1988), The compressible turbulent shear layer: an experimental study.

J. Fluid Mech. 197, p. 453.

S.K. LeLE (1994), Compressibility effects on turbulence. Ann. Rev. Fluid Mech. 26:211.

G. Lilley (1996), Personal communication. 
J. R. Ristorcelli (1997), A pseudo-sound constitutive relationship and closure for the dilatational covariances in compressible turbulence: an analytical theory. ICASE report 95-22, J. Fluid Mech. 347, p. 37.

J.R. RistoRCELLI (1997A), A closure for the compressibility of the source terms in Lighthill's acoustic analogy. ICASE report 97-44, submitted J. Fluid Mech. (1997).

J.R. RISTORCELLI (1997B), The pressure-dilatation covariance in compressible turbulence: validation of theory and modeling. ICASE report in progress.

J.R. Ristorcelli AND G. A. Blaisdell (1997), Consistent initial conditions for the DNS of compressible turbulence. ICASE Report 96-49, Phys. Fluids 9, p. 4.

S. SARKAR (1992), The pressure-dilatation correlation in compressible flows. Phys. Fluids A 12, p. 2674.

S. SARKAR (1995), The stabilizing effect of compressibility on turbulent shear flow. J. Fluid Mech. 282, p. 163.

A. Simone, G.N. Coleman And C. Cambon (1997), The effect of compressibility on turbulent shear flow: a rapid-distortion-theory and direct-numerical-simulation study. J. Fluid Mech. 330, p. 307.

E.F. Spina, A.J. Smits, S.K. Robinson (1994), The physics of supersonic boundary layers. Ann. Rev. Fluid Mech. 26:287.

S. Thangam, Y. Zhou, J.R. Ristorcelli. (1996), Analysis of compressible mixing layers using dilatational covariances model. AIAA-96-3076. 32nd AIAA/SAE/ASME/ASEE Joint Propulsion Conference, Lake Buena Vista, FL.

S. Thangam, J.R. Ristorcelli, Y. Zhou. (1996), Development and application of a dilatational covariances model for compressible mixing layers. AIAA-97-0723. 35th Aerospaces Sciences Meeting and Exhibit, Reno, NV.

W. A. VReman, N.D. Sandham AND K.H. Luo (1996), Compressible mixing layer growth rate and turbulence characteristics. J. Fluid Mech. 320, p. 235. 


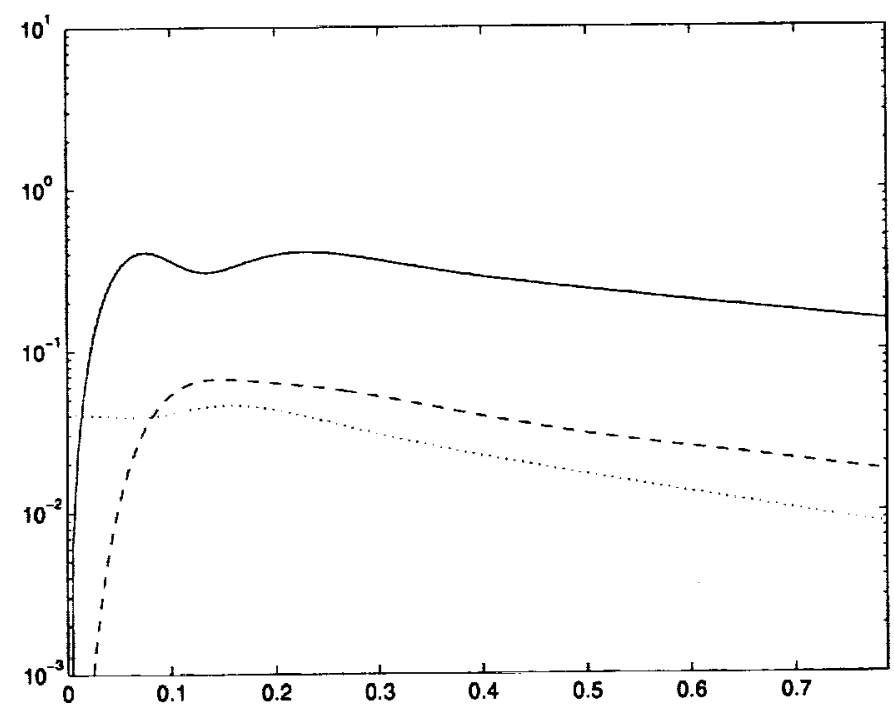

FIG. 1. Variance of the dilatation, $\langle d d\rangle$, for the isotropic decay as a function of time - three different initial conditions. IC1 - solid line; IC2 - dashed line; IC3 - dotted line.

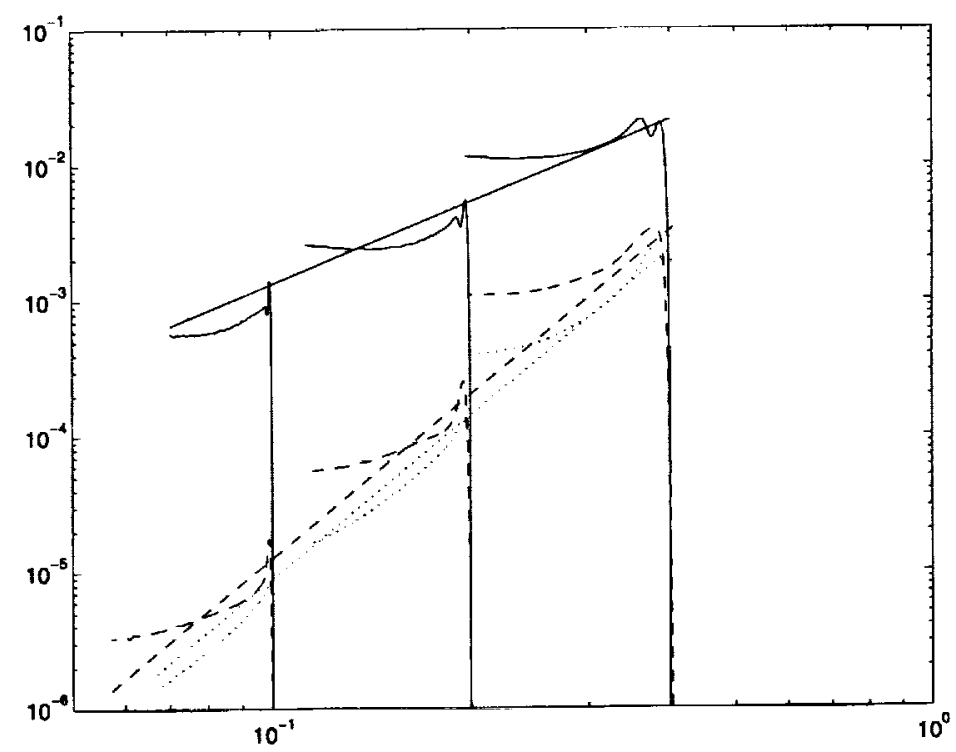

FIG. 2. Variance of the dilatation, $\langle d d\rangle$, versus $M_{t}$; three different Mach numbers - three different initial conditions. IC1 - solid line; IC2 - dashed line; IC3 - dotted line. 


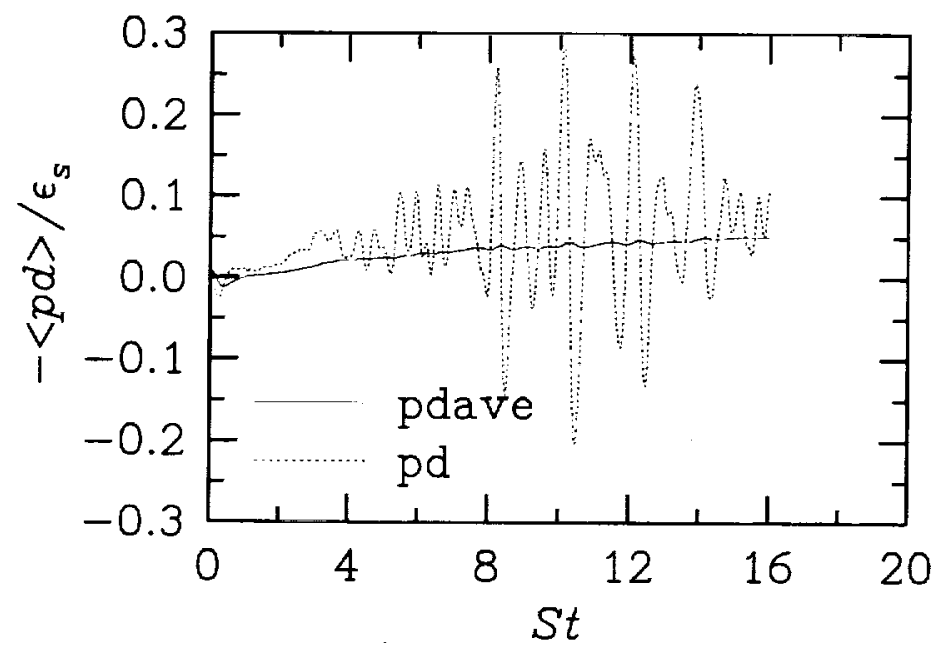

FIG. 3. The instantaneous and averaged pressure-dilatation-Blaisdell's rjr-s1 DNS.

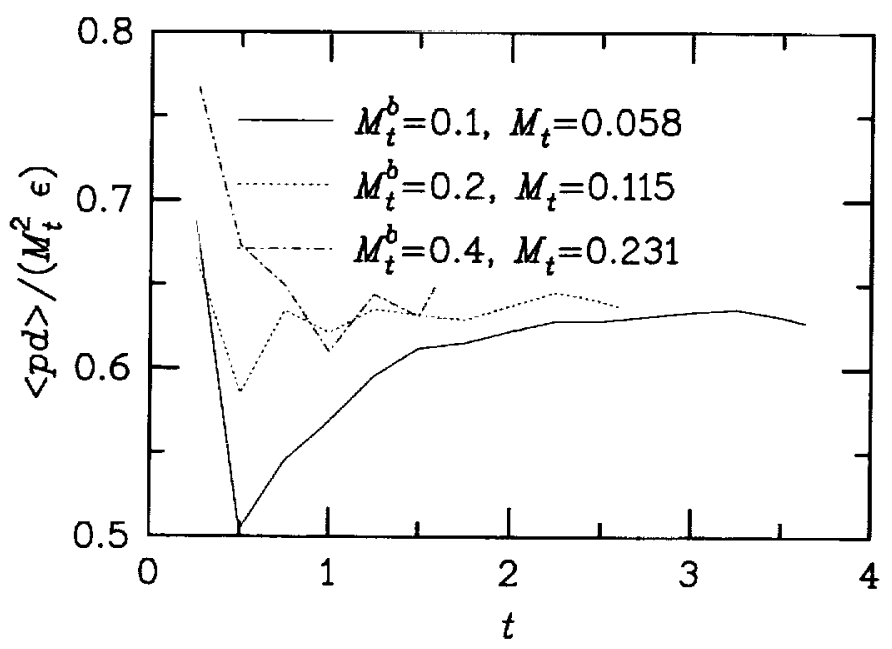

FIG. 4. Pressure-dilatation coefficient for the isotropic decay. 

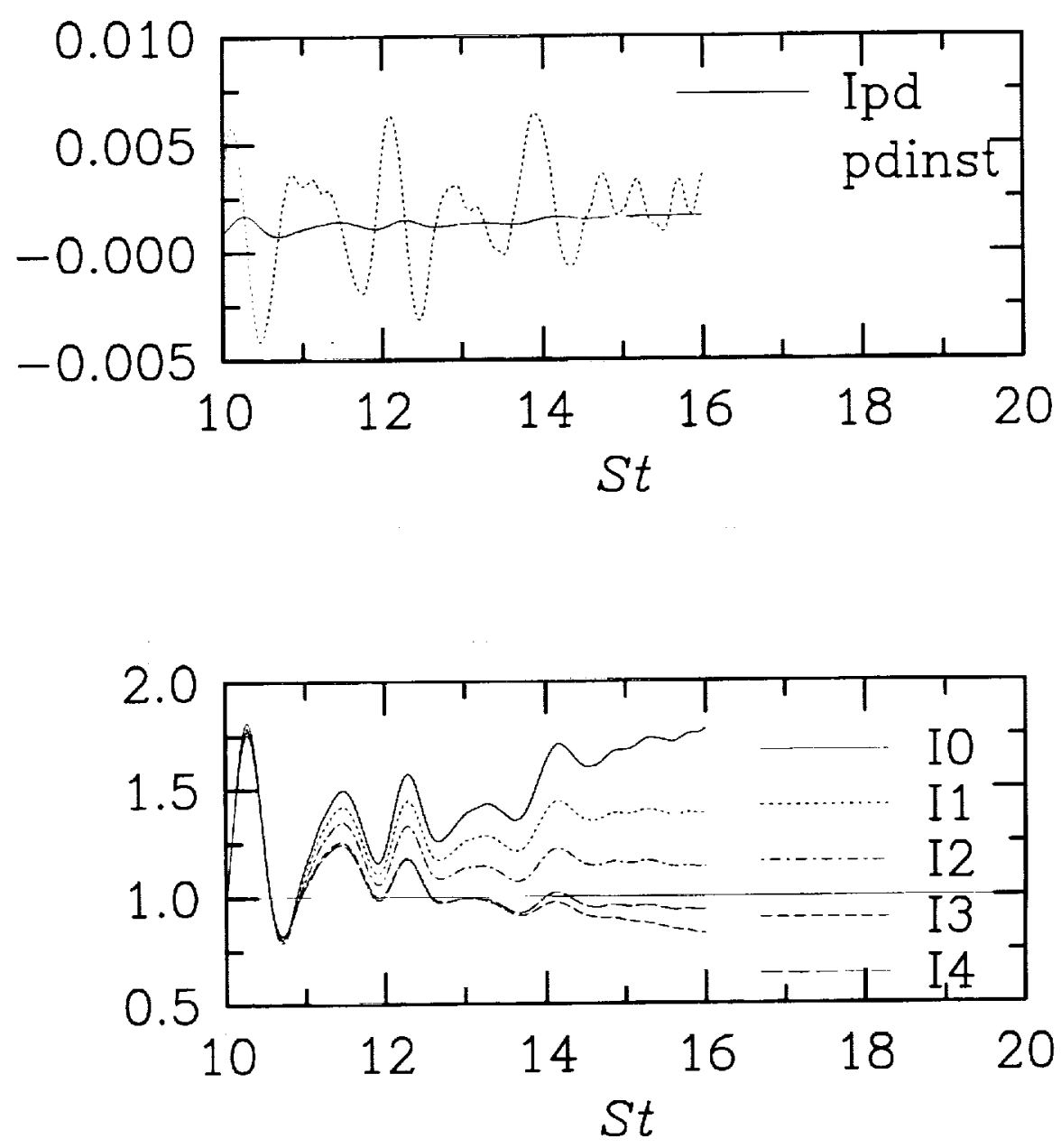

FIG. 5. Integral scalings for the pressure-dilatation in homogeneous shear. 


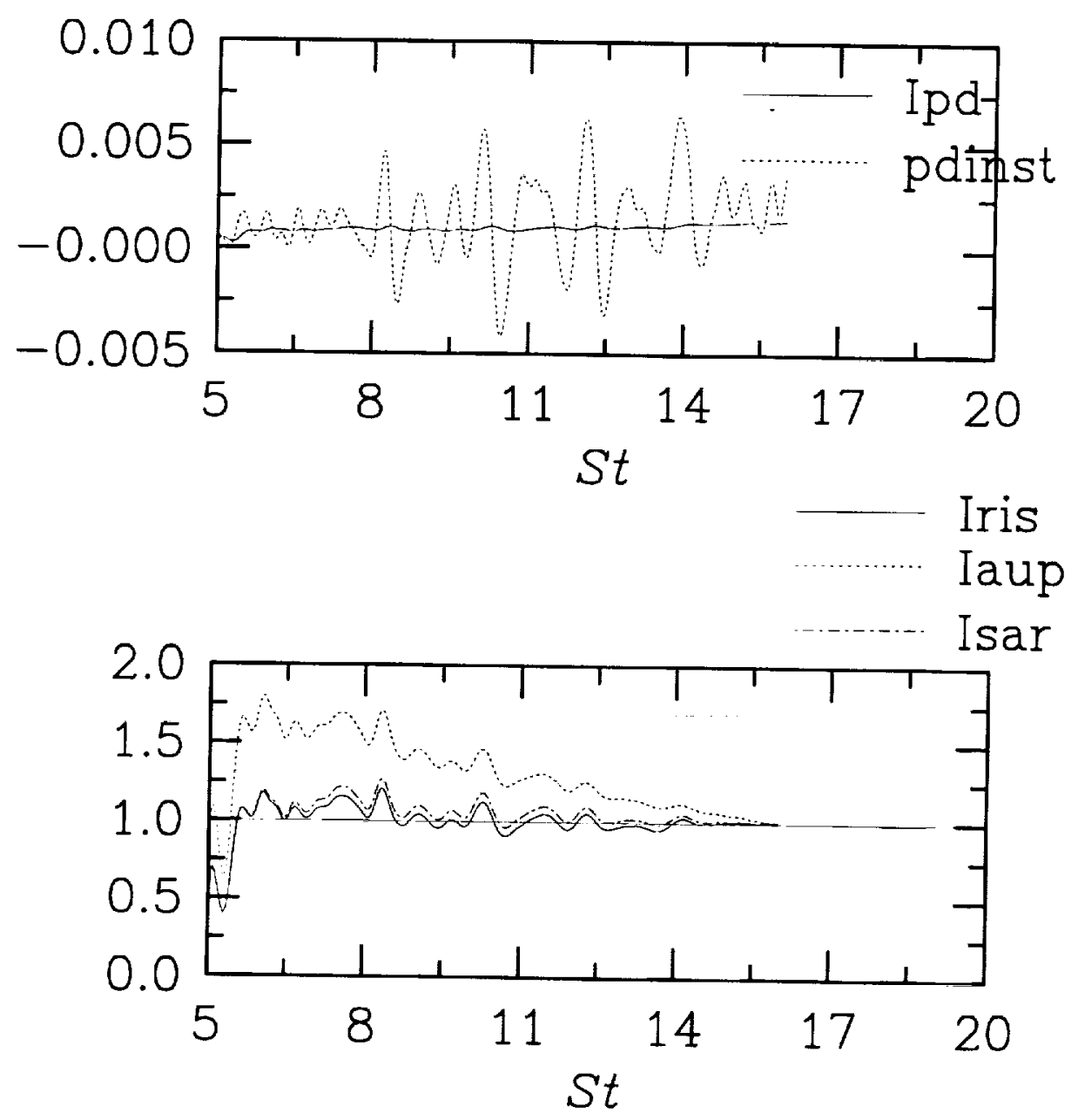

FIG. 6. Comparisons of the different models. 
Public reporting burden for this collection of information is estimated to average 1 hour per response, including the time for reviewing instructions, searching existing data sources. gathering and maintaining the data needed, and completing and reviewing the collection of information. Send comments regarding this bur Operations and Reports, 1215 Jefferson collection of information, including suggestions for reducing this burden, to Washington Headquareser. Paperwork Reduction Project (0704-0188), Washington, DC 20503.

Davis Highway, Suite 1204, Arlington, VA 22202-4302, and to the Office of Management and Budget, Paperwork Reduction Pro

\begin{tabular}{|l|c|c|}
\hline 1. AGENCY USE ONLY(Leave blank) & $\begin{array}{c}\text { 2. REPORT DATE } \\
\text { January } 1998\end{array}$ & $\begin{array}{c}\text { 3. REPORT TYPE AND } \\
\text { Contractor Report }\end{array}$
\end{tabular}

\section{TITLE AND SUBTITLE}

Some Results Relevant to Statistical Closures for Compressible

Turbulence

\section{FUNDING NUMBERS}

C NAS1-19480

WU 505-90-52-01

6. AUTHOR(S)

J. R. Ristorcelli

7. PERFORMING ORgANIZATION NAME(S) AND ADDRESS(ES)

Institute for Computer Applications in Science and Engineering

Mail Stop 403, NASA Langley Research Center

Hampton, VA 23681-0001

8. PERFORMING ORGANIZATION

REPORT NUMBER

ICASE Report No. 98-1

9. SPONSORING/MONITORING AGENCY NAME(S) AND ADDRESS(ES)

National Aeronautics and Space Administration

10. SPONSORING/MONITORING AGENCY REPORT NUMBER

Langley Research Center

Hampton, VA 23681-2199

NASA/CR-1998-206902

ICASE Report No. 98-1

\section{SUPPLEMENTARY NOTES}

Langley Technical Monitor: Dennis M. Bushnell

Final Report

To appear in Proceedings of Modeling Complex Turbulent Flows, by Kluwer Academic Press

12a. DISTRIBUTION/AVAILABILITY STATEMENT

12b. DISTRIBUTION CODE

Unclassified-Unlimited

Subject Category 34

Distribution: Nonstandard

Availability: NASA-CASI (301)621-0390

13. ABSTRACT (Maximum 200 words)

For weakly compressible turbulent fluctuations there exists a small parameter, the square of the fluctuating Mach number, that allows an investigation using a perturbative treatment. The consequences of such a perturbative analysis in three different subject areas are described: 1) initial conditions in direct numerical simulations, 2) an explanation for the oscillations seen in the compressible pressure in the direct numerical simulations of homogeneous shear, and 3) for turbulence closures accounting for the compressibility of velocity fluctuations.

Initial conditions consistent with small turbulent Mach number asymptotics are constructed. The importance of consistent initial conditions in the direct numerical simulation of compressible turbulence is dramatically illustrated: spurious oscillations associated with inconsistent initial conditions are avoided, and the fluctuating dilatational field is some two orders of magnitude smaller for a compressible isotropic turbulence. For the isotropic decay it is shown that the choice of initial conditions can change the scaling law for the compressible dissipation.

A two-time expansion of the Navier-Stokes equations is used to distinguish compressible acoustic and compressible advective modes. A simple conceptual model for weakly compressible turbulence - a forced linear oscillator is described. It is shown that the evolution equations for the compressible portions of turbulence can be understood as a forced wave equation with refraction. Acoustic modes of the flow can be amplified by refraction and are able to manifest themselves in large fluctuations of the compressible pressure.

14. SUBJECT TERMS

dilatation, compressible turbulence, turbulence modeling

15. NUMBER OF PAGES

28

16. PRICE CODE

$\mathrm{AO} 3$

\begin{tabular}{|l|l|l|l|}
\hline $\begin{array}{l}\text { 17. SECURITY CLASSIFICATION } \\
\text { OF REPORT } \\
\text { Unclassified }\end{array}$ & $\begin{array}{l}\text { 18. SECURITY CLASSIFICATION } \\
\text { OF THIS PAGE } \\
\text { Unclassified }\end{array}$ & $\begin{array}{l}\text { 19. SECURITY CLASSIFICATION } \\
\text { OF ABSTRACT }\end{array}$ & $\begin{array}{l}\text { 20. LIMITATION } \\
\text { OF ABSTRACT }\end{array}$ \\
\hline NSN 7540-01-280-5500 & & $\begin{array}{l}\text { Standard Form 298(Rev. 2-89) } \\
\text { Prescribed by ANSI Std. Z39-18 } \\
298-102\end{array}$ \\
\hline
\end{tabular}




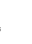

